\title{
Management of Distal Femoral Fracture with Distal Femoral Nail
}

\author{
Jabed Jahangir ${ }^{*}$ \\ Md lqbal Hossain ${ }^{1}$ \\ Md Myen Uddin Mojumder ${ }^{2}$ \\ Mohammad Hossain ${ }^{1}$ \\ Md Anwar Hossain ${ }^{1}$ \\ Md Imroz Uddin'
}

'Department of Orthopaedics Chittagong Medical College Chittagong, Bangladesh.

${ }^{2}$ Department of Orthopaedics Chittagong General Hospital Chittagong, Bangladesh.

\begin{abstract}
Background: For a long time distal femur fracture were considered difficult to heal and often led to a degree of disability. The incidence of malunion, nonunion and infection are relatively high in many reported series. The management goal of distal femur fractures are correction of axial alignment, length, and rotation, restoration of motion and rapid union so as to return the patient to normal function. We present here result of retrograde nailing of extra-articular (AO 33A1 to A3) fractures of the distal femur using Distal Femoral Nail (DFN). Methods: In Between 2008 to 2010 total 17 patients (One lost to follow up so 16 available for final assessment) underwent open reduction and internal fixation for distal femoral fracture in Chittagong Medical College Hospital and Private Hospital in Chittagong city. There were 13 male and 3 female patients, age range from 18 years to 70 years, mean age 42.75 years. Average length of follow-up was 17.5 months (12 months to 24 months). Results: Total 16 patients was available for follow up. Regular fracture healing was observed in 14 cases. Delayed union in 2 case. One patient had infection, and two patients had leg shorting of 0.5 and $1 \mathrm{~cm}$. Axial misalignment (Varus/valgus angulations) was found in 4 cases $\left(5^{\circ}-10^{\circ}\right)$ there were no implant failures. On the bases of a Leung score to measure functional outcome, 4 cases had excellent, 8 cases had good and 4 cases had fair result. Poor results were not seen in any patient. In this series 13 cases were satisfactory and 3 cases were unsatisfactory. Conclusion: Distal Femoral Nail is handsome tool to treat distal femoral fracture.
\end{abstract}

Key words: Distal Femur; Fracture; Distal Femoral Nail (DFN); Internal fixation.

\section{INTRODUCTION}

Distal femur fracture comprise $4-6 \%$ of all femoral fractures ${ }^{1,2}$. There is a bimodal distribution of distal femur fracture in general population, 15-50 years of age, predominantly in males, sustaining high-energy trauma, and 50+ years of age predominantly in females, with osteoporosis, who sustain relatively low energy trauma ${ }^{2}$. Biomechanically the quadriceps, adductors, hamstrings and gastrocnemius muscles can all cause displacement of the fracture fragments ${ }^{3}$. The gastrocnemius may cause posterior angulation of the distal fragment, overriding can caused by the combined action of the quadriceps and hamstrings. The heads of gastrocnemius may also rotate and spread intercondylar fractures. The adductors may cause varus or valgus deformities, depending on the fracture configuration and its relationship to the adductor tubercle. For a long time they were considered difficult to heal and often led to a degree of disability. These difficulties become greater when they are associated with elderly patients who present with a high degree of osteopenia. So when considering fixation, fixation system needs to be strong enough to resist these deforming forces and yet adaptable enough to deal with the various fracture patterns.

\section{Dr. Jabed Jahangir}

Assistant Professor

Department of Orthopaedics

Chittagong Medical College

Chittagong, Bangladesh.

Mobile : +8801711441285

E-mail : iitpmtr@gmail.com 
The popliteal artery is relatively fixed and lies in close relation to distal femur and can be damaged by a posteriorly angulated fracture, but this is rare, with an incidence of approximately $0.2 \% \%^{3}$. Another important consideration is regaining full knee motion and function which may be difficult because of the proximity of these fractures to the knee joint. The incidence of malunion, nonunion and infection are relatively high in many reported series ${ }^{4}$. The goals of management of distal femur fractures are correction of axial alignment, length, and rotation, restoration of motion and rapid union so as to return the patient to normal function ${ }^{5}$. The treatment of distal femoral fractures has undergone a substantial evaluation in the last three decades. Initial publication by Neer, Connolly, Mooney and Stewart and their colleagues conclude that non operative methods primarily traction alone or the combination with a cast, were superior to open reduction and internal fixation. But later work from AO (Arbeitsgemeinschaft fur Osteosynthesefragen) group and others with better fixation device and soft tissue handling it was clearly demonstrated that operative treatment fur superior to non operative one and has been abandoned as treatment for fractures of the distal femur at the end of 1960ies ${ }^{6}$. For operative treatment many different fixation methods have been described, Angled Blade Plate (ABP) Dynamic Condylar Screw (DCS) Condylar Buttress Plate (CBP) antegrade intramedullary nail, retrograde nail, flexible nail, Minimally Invasive Skeletal Stabilization (LISS) plate, external fixation, total knee replacement ${ }^{7}$. We investigated the feasibility and functional outcome of retrograde nailing of extra-articular (AO 33A1 to A3) fractures of the distal femur using Distal Femoral Nail (DFN).

\section{MATERIALS AND METHODS}

It is a prospective study conducted in Chittagong Medical College Hospital and Private Hospital in Chittagong city. Study period from July 2008 - June 2010. Total 17 patients irrespective of sex, with history of the fracture of distal third of femur AO type -A enrolled in the study. One patient lost to follow-up. So, Finally 16 patients available for evaluation. Inclusion criteria was age 18-70 years, fracture involving distal third of femur (AO type A) Closed fractures. Patient excluded if age below 18 years or above 70 years, Open fractures, Pathological fractures, Patients with medical problem eg. DM. Patients were managed according to standard protocol. After initial stabilization of patient complete history of the selected cases were taken. Complete local and general examination done. Radiological and general investigation done and patient was finally prepared for operative treatment. Proper counseling of the patients done regarding operative procedure, its merits and demerits, other surgical options, availability of treatment elsewhere, probable operative and post operative complications that may occur, possible post operative sequels. Informed written consent was taken from each case included in this study. All issues regarding patient's welfare was approved by the local ethical committee. All patients was operated under spinal anaesthesia and was receive prophylactic antibiotics.
Postoperatively limb was elevated on a pillow keeping the knee in $15-20^{0}$ flexion. The patient was started isometric quadriceps exercises after operation as patient tolerated. After 48 hours, drain was removed. The patient was allowed to move out of bed using crutches and passive range of motion was started. Stitches were removed on the $10^{\text {th }}$ to $14^{\text {th }}$ postoperative day. Patients was follow up clinically and radiologically at $6,12,18,24$ weeks. Evaluation of the final outcome was based on the Leung score for distal femoral fractures.

\section{RESULTS}

This was a clinical trial carried out at Chittagong Medical College Hospital and Private Hospital Chittagong city from July 2008 to June 2010 involving 17 patients with distal femoral fractures. One patient lost to follow up so ultimately 16 patients available for final assessment. Each patient was treated by open reduction and internal fixation by Distal Femoral Nail (DFN). Average length of follow-up was 17.5months (12 months to 24 months).

Table 1 : Age and sex distribution of study $(n=16)$.

\begin{tabular}{lccccc}
$\begin{array}{c}\text { Age group } \\
\text { (Years) }\end{array}$ & $\begin{array}{c}\text { Number } \\
\text { of } \\
\text { patients }\end{array}$ & Percentage & $\begin{array}{c}\text { Average } \\
\text { age } \\
\text { (Years) }\end{array}$ & Male & Female \\
\hline $18-29$ & 03 & $18.75 \%$ & & 03 & 00 \\
$30-39$ & 06 & $37.50 \%$ & & 06 & 00 \\
$40-49$ & 02 & $12.50 \%$ & & 02 & 00 \\
$50-59$ & 02 & $12.50 \%$ & 42.75 & 00 & 02 \\
$60-70$ & 03 & $18.75 \%$ & & 02 & 01 \\
\hline
\end{tabular}

There were 13 male and 3 female patients, age range from 18 years to 70 years, mean age 42.75 years. Out of 16 patient's $3-$ drivers, 2-day worker, 2- business man, 2-service holder, 3students and 3-house wife, others-1.

Table 2 : Mechanism of injury.

$\begin{array}{lcr}\text { Mechanism Number of patients } & \text { Percentage } \\ \text { High energy trauma } & 12 & 75.00 \% \\ \text { Motor vehicle accident } & 07 & 43.75 \% \\ \text { Motor cycle accident } & 02 & 12.50 \% \\ \text { Auto-pedestrian accident } & 02 & 12.50 \% \\ \text { Fall from height } & 01 & 6.50 \% \\ \text { Low energy trauma } & & \\ \text { (Fall from bed, chair) } & 04 & 25.00 \%\end{array}$

There were 11 patients involving right side and 5 patients involving left side. There was 7 AO type A1 fractures, $6 \mathrm{AO}$ type-A2 fractures, $3 \mathrm{AO}$ type-A3 fractures. 
Table 3 : Time and Rate of healing.

\begin{tabular}{lrrr}
$\begin{array}{l}\text { Time of union } \\
\text { (Weeks) }\end{array}$ & No. of patients & Percentage & $\begin{array}{r}\text { Union rate } \\
\text { (Regular) }\end{array}$ \\
12 & 02 & $12.50 \%$ & \\
14 & 04 & $25.00 \%$ & \\
15 & 08 & $50 \%$ & $87.50 \%$ \\
25 & 01 & $6.50 \%$ & \\
28 & 01 & $6.50 \%$ & \\
\hline
\end{tabular}

Regular fracture healing (Formation of circumferential bridging callus across the fracture allowing full weight bearing) was observed in 14 cases. Delayed union (Union time over 24 weeks) was observed in 2 case. Fracture consolidation occurred after an average time of 15.81 weeks (Range 12-28 weeks).

Table 4 : Range of knee motion.

\begin{tabular}{lcc} 
Range of knee motion & No. of cases & Percentage \\
$130-140$ & 01 & $6.25 \%$ \\
$120^{\circ}-130^{\circ}$ & 02 & $12.50 \%$ \\
$110^{\circ}-120^{\circ}$ & 07 & $43.75 \%$ \\
$90^{\circ}-110^{\circ}$ & 05 & $31.25 \%$ \\
$75^{\circ}-90^{\circ}$ & 01 & $6.25 \%$ \\
\hline
\end{tabular}

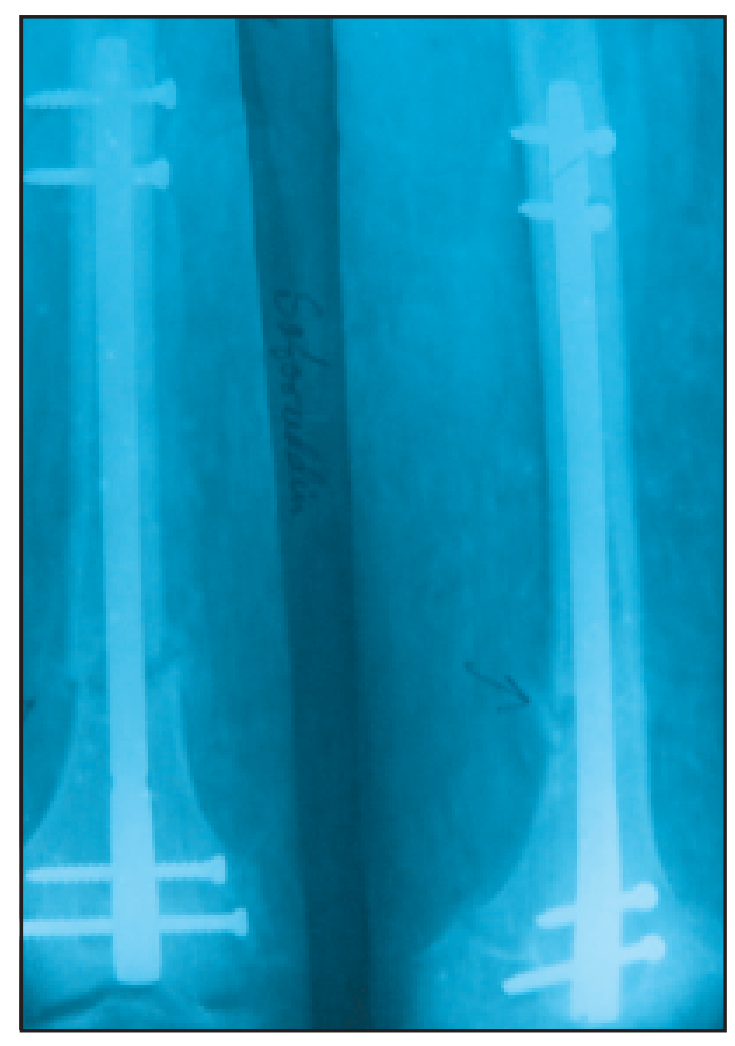

Image 1 : Distal femoral fracture.
Although there were no problem with the wound healing in any patients, two patients had a delayed union, one patient had infection, and two patients had leg shorting of 0.5 and $1 \mathrm{~cm}$. Axial misalignment (Varus/valgus angulations) was found in 4 cases $\left(5^{\circ}-10^{\circ}\right)$ there were no implant failures. One patient present with wound infection at first follow-up visit. He was treated first with antibiotic according to culture sensitivity. But there was repeated collection, so, debridement done. Patient was ultimately infection free at 16 weeks with delayed union at $28^{\text {th }}$ weeks. On the bases of a Leung score to measure functional outcome, 4 cases had excellent, 8 cases had good and 4 cases had fair result. Poor results were not seen in any patient. In this series 13 cases were satisfactory and 3 cases were unsatisfactory.

\section{DISCUSSION}

Retrograde intramedullary nailing was developed in an attempt to overcome the limitations of antegrade nailing. Distal femur fracture where maintaining alignment is difficult with antegrade nailing, in morbid obese patients, fracture involving ipsilateral femur and tibia fracture, multi-system injuries (Necessity of extension table) or distal fracture type and to ensure the advantages of minimal invasive technique in contrast to plate osteosynthesis. While retrograde nailing is an accepted minimally invasive procedure for osteosynthesis of femoral fractures, it's particularly recommended for type 33 A1 to A3 and $\mathrm{C} 1$ fractures according to the $\mathrm{AO}$ classification ${ }^{8}$. In the present series, the age of the patients varied from minimum 18 years to maximum 70 years, mean age being 42.75 years. Similar findings were also been noted by Seifert et al, Leung et al and Gellman et al were 44 years (Range 17-92 years) 46.5 years (Rang 22-85years) and 50 years (Range 26-84 years) respectively ${ }^{9-11}$. The mechanism of injury and the bimodal distribution of high energy fractures occurring in younger males and the low energy fractures in elderly females were comparable with previous studies. Period of fracture healing was average 17.5 weeks (Range 8-68 weeks) in the series of Handolin et al, average 12 weeks (Range 8-24 weeks) in Leung et al series and average 12.5 weeks (Range 8-16 weeks) in the series of Gellman et al ${ }^{12,10,11}$. Average healing time in our series was 15.81 weeks (Range 12-28 weeks). 87.50 percent of the fracture were united in this series and were capable of full weight bearing. 100 percent of the fractures were healed in the series of Seifert et al, Gellman et al and Grass et al ${ }^{9,11,13}$. But in the in series of Handolin et al 95 percent of the fractures were healed in regular healing time (Less than 24 weeks) ${ }^{12}$. No nonunion occurred in present series. Similar finding were found in the series of Seifert et al, Grass et al and Gellman et al ${ }^{9,13,11}$. But Ostrum et al, Ricci et al, Handolin et al found nonunion of $6 \%, 6 \%$ and $4.7 \%$ cases respectively in their series ${ }^{14,15,12}$. Delayed union occurred in 2 patients $(12.50 \%)$ in this series and ultimately healed. Similar finding was found in the series 
of Handolin et $\mathrm{al}^{12}$. But no delayed union occurred in the series of Seifert et al, Grass et al and Leung et $\mathrm{al}^{9,13,10}$. Regarding range of knee motion, at the latest follow-up examination was $75^{\circ}$ to $90^{\circ}$ in 1 case, $90^{\circ}$ to $110^{\circ}$ in 5 cases, $110^{\circ}$ to $120^{\circ}$ in 7 cases, $120^{\circ}$ to $130^{\circ}$ in 2 cases and $130^{\circ}$ to $140^{\circ}$ in 1 case. The mean range was $115^{\circ}$. In the series of Grass et al average knee motion was $120^{\circ}$ and in the series of Gellman et al it was $106^{13,11}$. Axial mal-alignment (Varus-valgus and anteroposterior angulation) was found radiologically in $18.75 \%$ cases in this series. In the series of Grass et al, and Handolin et al it was $17 \%$, and $4.3 \%$ respectivelly ${ }^{13,12}$. But Seifert et al did not find any axial malalignment ${ }^{9}$. There was one infection in this series. No infection was found in the series of Leung et al, Seifert et al and Grass et $\mathrm{al}^{9,13,10}$. In the final follow-up, the satisfactory result (Excellent and good) of this series was $75.00 \%$ These results were similar to the study conducted by Seifert et al and Gellman et al where was $84 \%$ and $81.7 \%$ respectively ${ }^{9,13}$. But in the series of Leung et al satisfactory result was $94 \%{ }^{10}$.

\section{CONCLUSION}

Successful management of distal femoral fracture is possible with adherence to the basic principle of anatomic reduction, stable fixation, and early motion. In conclusion, our results suggest that the DFN is a reliable when options in the treatment of distal femoral fractures are considered. Despite the fact that different surgeons in the start of their learning curves performed the operation in this series, the average clinical and radiological results were encouraging. Union of the fracture was achieved in our series in most cases within reasonable time, and complication rate was fairly low. The simplicity of this method also facilitates fracture fixation in the patients with multiple traumas. However, little is known about the longer effects of trans-articularly inserted retrograde femur implants. So long terms follow up and large-scale study is needed to evaluate the final effect of this procedure.

\section{DISCLOSURE}

All the authors declared no competing interest.

\section{REFERENCES}

1. Kolmert L, Wulff K. Epideiology and treatment of distal femoral fractures in adults. Acta Orthop Scand. 1982; 53: 957-962.

2. Martinet O, Cordey J, Harder Y. The epidemiology of fractures of the distal femur. Injury. 2000; 31(3): 62-63.

3. Stewart M J, Sisk T D, Wallace S L. Fractures of the distal third of the femur: A comparison of methods of treatment. J Bone Joint Surg Am. 1966; 48:784-807.

4. Wgittle, P A., and George, W. Wood II. Fractures of Lower Extremity, in S T. Canale, K Daugherty and L Jones (editors) $10^{\text {th }}$ ed, Cambell's operative orthopaedics. Philadelphia:Mosby. 2003;3: 2805.

5. Schatzker J, Tile M. The Rationale of Operative Fracture Care .New York: Springer-Verlag. 1987.

6. Templeman D C. Fractures of the distal femur, in R.B. Gustilo, F. Richard, and D C. Templeman (eds), Fractures and Dislocation. Philadelphia: Mosby. 1993;2:981.

7. Foster M C, Komarsamy B, Davision J N. Distal femoral fracture: A review of fixation methods. Injury. 2006; 37: 97-108.

8. Macrae R, Esser M. Practical fracture treatment. $3^{\text {rd }}$ ed. London: Churchil Livingstone. 2002.

9. Seifert J, Stengel D, Matthes G, Hinz P, Ekkerkamp A, Ostermann A W. Retrograde fixation of distal femoral fractures;results using a new nail system. J Orthop Trauma. 2003; 17(5):488-495.

10. Leung K S, Shen W Y, Mui L T, Grosse A. Interlocking intramedullary nailing for supracondylar and intercondylar fractures of the distal part of the femur. J Bone Joint surg Am. 1991; 73(3):332-340.

11. Gellman R E, Paiement G D, Green H D, Coughlin R R. Treatment of supracondylar femoral fractures with a retrograde intramedullary nail. Clinical Orthopaedics and Related Research. 1996; 332: 90-97.

12. Handolin L, Pajarinen J, Lindahl J, Hirvensalo E. Retrograde intramedullary nailing in distal femoral fractures-result in a series of 46 consecutive operations. Injury. 2004; 35:517-522.

13. Grass R, Biewener A, Rammelt S, Barthel S, and Zwipp H .Clinical trial with the distal femoral nail. Unfallchirurg. 2002; 105(7): 587-594.

14. Ostrum R F, DiCicco J, Lakatos R, Poka A. Retrograde intramedullary nailing of femoral diaphyseal fractures. J Orthop Trauma. 1998; 12(7):464-468

15. Ricci W M, Bellabarba C, Evanoff B, Herscovici D, DiPasquale T, and Sanders R. Retrograde versus antegrade nailing of femoral shaft fractures. J Orthop Trauma. 2001; 15(3):161-169. 